\title{
Carcinoma Espinocelular: Prevención y Diagnóstico Precoz en Odontología
}

\author{
SquamousCellCarcinoma: PreventionAndEarlyDiagnosisInDentistry
}

\section{Resumen}

El carcinoma espinocelular (CEC) es la neoplasia de mayor prevalencia en la cavidad oral. Debido a que la mayoría de estos casos son diagnosticados tardíamente, el tratamiento ha sido relacionado a cirugías radicales y deformantes, así como a altas tasas de recidiva y pronósticos poco promisores.

Actualmente, gracias a los avances tecnológicos, son realizados tratamientos quirúrgicos más conservadores. Además, nuevos esquemas de quimio y radioterapia afectan significativamente el tratamiento y la evolución de los pacientes. No obstante, algunos investigadores han relatado apenas una mejora modesta en la tasa de sobrevida del CEC en estado avanzado, el mismo que continua presentando un pronóstico extremadamente pobre.

Casos iniciales de CEC tienen mejor pronóstico cuando comparados a casos avanzados de la enfermedad. Por tanto, la prevención, un diagnóstico precoz y el establecimiento de un tratamiento oportuno siguen siendo esenciales para prevenir muertes prematuras, desfiguramiento estético y sequelas funcionales en nuestros pacientes.

Palabras clave:

\section{Abstract}

Squamous cell carcinoma (SCC) is the most prevalent malignancy in the oral cavity. Because most of the SCC cases are diagnosed late, treatment has been linked to radical and disfiguring surgery, as well as high rates of recurrence and unfavorable forecasts.

Currently, technological advances allow more conservative surgical treatments. In addition, new models of chemotherapy and radiotherapy significantly affect treatment and outcome of patients. However, some researchers have reported only a small improvement in the survival rate in advanced SCC, the same that continues presenting an extremely poor prognosis.

SCC initial cases have a better prognosis when compared to advanced cases. Therefore, the prevention, early diagnosis and prompt treatment setting are essential to prevent premature deaths, aesthetic disfigurement and functional sequels caused by this disease.

Keywords:

\section{Introducción}

El carcinoma de orofaringe es la sexta causa más común de malignidad en el mundo. Entre estos tipos de cáncer, el carcinoma espinocelular bucal (CEC) es el más frecuente, representando aproximadamente el 95\% de los tumores malignos en esta región (Carvalho et al., 2001).

El CEC representa un problema grave de salud pública con una tendencia al crecimiento, en particular en los países emergentes (Sankaranarayanan et al., 1998). Así, en la India representa la tercera parte de todas las neoplasias malignas, mientras que en Brasil entre $3-5 \%$, siendo considerado uno de los diez tipos más comunes de cáncer (INCA-Brasil, 2007). Nuevos esquemas quirúrgicos y terapéuticos han sido utilizados con relativo éxito en niveles de atención secundaria y terciaria. Sin embargo, esos recursos se destinan a tratar estadios ya avanzados de la enfermedad, lo que supone un elevado riesgo de mal pronóstico para el paciente y un elevado costo para los servicios de salud pública.

Una mayor tasa de supervivencia, mejor calidad de vida, menos cirugías deformantes y menores costos de tratamiento están directamente relacionados con el diagnóstico temprano de la enfermedad (Scully et al., 2006). Por lo tanto, la prevención y el diagnóstico precoz siguen siendo los principales recursos para el combate efectivo del CEC (Sklenicka et al., 2010).

El objetivo del presente artículo es realizar una revisión concisa de la epidemiologia, de los criterios diagnósticos y de las medidas de prevención del CEC oral.

\section{Casos Clínicos}

\section{José Luis Muñante Cárdenas'; Luis Augusto Passeri²}

1 Magister en Cirugía e Traumatología BucoMaxilo-Facial. Doctorando en Cirugía. Universidad Estatal de Campinas, DDS, MSc.

2 Profesor Titular de Cirugía Buco-Maxilo-Facial, Área de Cirugía Plástica. Departamento de Cirugía, Facultad de Ciencias Médicas. Universidad Estatal de Campinas, DDS, MSc, PhD.

\section{Correspondencia:}

Dr. Luis Augusto Passeri: Rua. Tessália Vieira de Camargo

126, Ciudad Universitária "Zeferino Vaz" - Campinas - SP - Brasil - Código postal: 13083-887.

Departamento de Cirugía - Teléfono: 55-193521.9450

Fax: 55-19-3521.8043. email: passeri@fcm.unicamp.br

Fecha de recepción: 08 de abril

Fecha de aceptación: 14 de junio

\section{Metodología}

Presentamos dos casos clínicos sobre el manejo de CEC. Además, realizamos una revisión de artículos científicos publicados en inglés y portugués. Para este fin se utilizaron los buscadores Pubmed y Medline. Incluimos publicaciones en las que aparecieron como palabras clave "Cáncer Oral”, "Oral cancer prevention", "Oral Squamous Cell Carcinoma" y "Carcinoma espinocelular". Fueron consideradas revisiones sistemáticas, estudios prospectivos longitudinales y estudios retrospectivos. Veinticinco publicaciones sobre epidemiologia, criterios diagnósticos y medidas de prevención del CEC oral fueron consideramos de interés para este articulo. Se excluyeron los artículos sin resumen disponible y los casos clínicos. 


\section{Casos clínicos}

\section{Caso 1}

Paciente de género masculino, raza blanca, 74 años de edad, acude a la consulta por disfagia, con aproximadamente 6 meses de evolución. Paciente hipertenso en tratamiento. Niega otra patología sistémica. Consumidor crónico de alcohol y tabaco hace 45 años. Relata que la primera consulta fue con un Otorrinolaringologo, quien diagnosticó faringitis; además derivó al paciente para evaluación y tratamiento odontológico por "úlcera en lengua”. El odontólogo prescribió amoxicilina 500 mg x 7 días y enjuagatorios bucales. Dos meses mas tarde, y al no observar mejora del cuadro, refirió al paciente a nuestra consulta.

Al examen físico intra-oral se observó úlcera de de $2 \times 2 \mathrm{~cm}$ en borde lateral posterior derecho delengua, moderadamente dolorosa a la palpación, ligeramente sangrante, con bordes elevados, irregulares y endurecidos (Figura 1). No fueron detectados linfonodos palpables durante el examen clínico regional de cabeza y cuello. Fue realizada una biopsia incisional de la lesión y el espécimen fue enviado para análisis histopatológico. El diagnostico fue de Carcinoma espinocelular altamente diferenciado.

\section{Caso 2}

Paciente de género masculino, mestizo, 55 años, fue referido a consulta por odontólogo con "diagnostico clínico de granuloma piogénico.". Sin antecedentes de consumo de alcohol o tabaco. Paciente no sabía precisar el tiempo de evolución de la lesión, afirmando haber notado la presencia de esta solamente después de su crecimiento, hace 8 meses aproximadamente. Durante el examen físico intra-oral se constató a presencia de una lesión exofítica, sésil, localizada en mucosa palatina izquierda, presentando contorno y superficie regular, consistencia dura, bordes irregulares, cerca de $3,5 \mathrm{~cm}$ de extensión, áreas eritoplásicas, sangrante y con sintomatología dolorosa a la palpación (Figura 2). No fueron detectados linfonodos palpables durante el examen físico regional de cabeza y cuello. Fue realizada una biopsia incisional y el espécimen enviado para análisis histopatológico.El diagnóstico fue de Carcinoma espinocelular indiferenciado.

Ninguno de los pacientes presentó antecedentes familiares de cáncer. Después del diagnóstico, ambos fueron encaminados para la especialidad de cirugía de cabeza y cuello para iniciar su tratamiento.

\section{Discusión}

El CEC es la neoplasia mas común de la cavidad oral, siendo responsable por cerca del 95\% de las neoplasias de esta región (Carvalho et al., 2001). El CEC se origina en el tejido epitelial de revestimiento a partir de células de la capa basal, proliferando en dirección al tejido conjuntivo subyacente (Neville et al., 2002).

La causa del CEC es multifactorial. No existe un agente causal aislado claramente definido o aceptado. Los factores de riesgo comúnmente asociados son el consumo de alcohol y el tabaco. Estos factores son altamente sinérgicos y representan el 75\% de los cánceres de cavidad oral y orofaringe (Mansour et al., 2003; Scully et al., 2006). Otros factores también pueden ser considerados como agentes cancerígenos: la radiación ultravioleta (específicamente para cáncer de labio), la deficiencia de vitaminas $\mathrm{A}, \mathrm{C}$ o $\mathrm{E}$, defectos inmunes $\mathrm{y}$ virus, principalmente papiloma virus (Scully et al., 2006). Dentro de los factores de riesgo locales han sido señalados pobre higiene oral, defectos en superficies dentarias, por traumatismos o caries y dispositivos protéticos en mal estado (Rosenquist, 2005).

La razón de prevalencia entre hombre/ mujer es de 3:1. Esto puede ser explicado por una mayor asociación entre el consumo de alcohol y de tabaco entre los hombres. Sin embargo, esta proporción viene disminuyendo, probablemente debido a la adopción de estos hábitos también por las mujeres (Leão et al., 2005; Neville et al., 2002). En relación a la raza y edad, una mayor incidencia ha sido reportada en pacientes de raza blanca de más de 40 años. Estudios muestran más de $50 \%$ de los casos reportados en pacientes entre 45 y 65 años (Macfarlane et al., 1992; Neville et al., 2002).

\section{Aspectos clínicos y Iocalización}

El CEC se puede presentar clínicamente como ulceraciones o lesiones leucoplásicas, eritroplásicas o eritroleucoplásicas; lesiones exofíticas/endofiticas de superficie verrugosa, costrosa o granular que presentan un crecimiento lento. Los bordes suelen ser irregulares, endurecidos y elevados. Estas lesiones son típicamente asintomáticas. Sin embargo, en las fases más avanzadas de la enfermedad (donde ocurre invasión profunda de las estructuras anatómicas) el dolor -que puede ser leve o agudo e irradiado hacia el oído- y la disfagia pueden ser quejas notorias (Neville et al., 2002; Silva et al., 2004).

El CEC de lengua es el más común (40-60\%), siendo su pronóstico más pobre cuando comparado con otras localizaciones anatómicas en la cavidad oral (Zelefsky, 1992). Otros sitios frecuentemente afectados son el piso boca (15-20\%), las encías, reborde alveolar, paladar blando, paladar duro, área retromolar y la mucosa oral. Todos ellos representan entre 10 y 40\% (Zelefsky et al., 1992; Leemans et al., 1994). Los CEC de labio también son frecuentes y, por su localización externa, deberían ser fácilmente diagnosticados en una fase más temprana por Odontólogos (Figura 3).

\section{Diseminación de la enfermedad}

La diseminación metastásica del CEC ocurre largamente a través de los linfáticos para los linfonodos cervicales. Los primeros linfonodos a ser comprometidos son los submandibulares o los yugolodigástricos (Neville, 2002; RegeziSciubba, 2008) (Figura 4).

Si bien el CEC es generalmente considerado una enfermedad regional, la posibilidad de una metástasis a distancia debe ser muy tomada en cuenta. Estas afectan generalmente a los pulmones (66\%), huesos (22\%) e hígado (9.5\%). Otros órganos como riñón, glándula suprarrenal y cerebro también pueden ser afectados (Neville, 2002; RegeziSciubba, 2008). La probabilidad de metástasis a distancia es menor cuando el diagnóstico es realizado precozmente $(2 \%-3 \%)$, aumentando significativamente $(10 \%-40 \%)$ si el paciente es diagnosticado en un estadio ya avanzado de la enfermedad (Betka et al., 2001).

\section{Diagnostico diferencial}

Cuando el carcinoma bucal se presenta en su forma clínica típica de úlceras crónicas no cicatrizadas, otras condiciones ulcerativas deben ser consideradas. De hecho, puede resultar difícil diferenciar clínicamente el CEC de otras patologías con manifestaciones orales como TBC, micosis profundas (histoplasmosis, paracoccidiomicosis) o sífilis. Incluso le- 
siones traumáticas crónicas deben ser consideradas (Regezzi-Sciubba, 2008). Una cuidadosa anamnesis, historia clínica y estudio histopatológico son especialmente importantes para orientar el diagnóstico y el tratamiento inicial del paciente.

\section{Tratamiento}

Los CEC son tratados mediante la escisión quirúrgica de la lesión con márgenes de seguridad. La remoción quirúrgica concomitante de los ganglios linfáticos comprometidos puede ser indicada. La aplicación de radioterapia y quimioterapia puede ser establecida como tratamiento complementar (Cooper et al., 2004).

A pesar de los avances logrados en el tratamiento del cáncer localmente avanzado de cabeza y cuello, la supervivencia de los pacientes no ha aumentado significativamente en las últimas dos décadas.La enfermedad sigue presentando tasas inaceptablemente altas de recidivas (Sklenicka et al., 2010). En general, la supervivencia a los 5 ańos llega a cerca de 45 a 50\%. Después que metástasis regionales se hayan producido, la supervivencia disminuye a la mitad (Shiboski et al., 2005). En cambio, si una neoplasia pequeńa y localizada es diagnosticada y tratada oportunamente, el índice de cura a 5 años puede alcanzar de 60 a 70\% (y en las lesiones de labio inferior, el índice puede llegar hasta 90\%) (Parise Jr., 2000). Debido a estos resultados, autores coinciden en afirmar que cuanto mas rápido sea realizado el diagnóstico e iniciado el tratamiento, mayor será la probabilidad de cura (Altdestein et al., 2003).

\section{Rol del odontólogo en el diagnóstico precoz del cáncer oral}

Un alto porcentaje de profesionales de la salud (91\%), consideraron que los odontólogos juegan un papel muy importante en la prevención del cáncer oral. Sin embargo, solamente un tercio de los odontólogos encuestados se sintió en la capacidad de diagnosticar adecuadamente una lesión pre maligna o maligna en la cavidad oral (Clovis et al., 2002; Macpherson el al., 2003; Leão et al., 2005). Debido a estas situaciones, no es sorprendente encontrar que del 40 a $60 \%$ de los casos sean diagnosticados ya en etapas avanzadas $(43 \%$ con metástasis regionales y un $17 \%$ con metástasis a distancia) y por lo tanto, con mal pronóstico.
Esta realidad también puede observarse en los casos presentados. En el caso No1 por ejemplo, una úlcera sangrante en borde lateral de lengua (uno de los locales mas comunes de presentación del CEC (Zelefsky, 1992), fue manejada de manera negligente, perdiéndose varios meses antes de establecerse un diagnóstico. Del mismo modo, disfagia, dificultad de movimiento de la mandíbula y dolor irradiado hacia el oído son síntomas muy comunes en CEC avanzados (Neville et al., 2002). Otro hecho que pasó inadvertido en este caso fue que el paciente era consumidor crónico de alcohol y tabaco de larga data, factores de riesgo fuertemente ligados al cáncer oral (Mansour et al., 2003; Rosenquist, 2005; Scully et al., 2006) y que también debieron alertar a los profesionales durante la anamnesis y evaluación física. En el caso No2 nuevamente se observa una deficiencia en la identificación de lesiones. El "diagnostico clínico" realizado por el profesional no alertó al paciente sobre la necesidad de buscar atención inmediata, retrasando innecesariamente el inicio del tratamiento.

Como podemos observar en estos dos casos, un factor importante que influyó negativamente en el establecimiento del diagnóstico fue la demora delos pacientes en procurar atención profesional. El comportamiento asintomático de las lesiones iniciales malignas parece explicar esta conducta (Macpherson et al., 2003). Pero también existen otras variables que aparentemente contribuyen con esta demora: estado socioeconómico y cultural, género, dificultad de reconocer la "malignidad" de la lesión y a la dificultad de acceder de manera rápida a los servicios públicos de salud (Fang, 2002).

\section{Medidas de prevención}

La medida más importante de prevención esta representada por la evaluación física intraoral, un procedimiento simples, rápido, no invasivo y económico. Este procedimiento es tan efectivo que ha demostrado una sensibilidad superior a $60 \%$ en la detección precoz del CEC. Cuando practicado en pacientes con riesgo elevado, principalmente consumidores de alcohol y tabaco, puede reducir la mortalidad en hasta $32 \%$ (Silva et al., 2004). Según Leão et al. (2005), odontólogos que invirtieron un tiempo superior a la media de las consultas durante la evaluación inicial de sus pacientes, tuvieron mayor posibilidad de detectar lesiones orales sospechosas.
Diversas estrategias de prevención pueden ser adoptadas, pero todas ellas deben estar siempre orientadas a conseguir tres objetivos principales (Kuriakose, 2006): 1) Informar permanentemente a nuestros pacientes sobre la alta relación entre cáncer y hábitos nocivos para la salud, como el consumo de tabaco y alcohol o la sobre exposición a la radiación solar; 2) Reconocer tempranamente lesiones pre malignas, estableciendo las medidas terapéuticas necesarias para evitar la evolución de la enfermedad; y 3) Realizar diagnóstico precoz de CEC, reduciendo así la morbilidad del tratamiento.

\section{Conclusiones}

La prevención juega un papel muy importante en la lucha contra el cáncer. La falta de un diagnóstico oportuno puede retrasar el inicio del tratamiento, permitiendo metástasis de la enfermedad. Esto puede traer consecuencias directas sobre la complejidad, costo del tratamiento y principalmente sobre el pronóstico de la enfermedad. El odontólogo, mismo no siendo especialista en cirugía maxilofacial o en patología oral, es por su área de actuación el profesional de la salud llamado a estar en la primera línea de defensa contra el CEC. Por tanto debe estar en la capacidad de diagnosticar de forma adecuada y oportuna lesiones pre malignas y malignas de la cavidad oral.

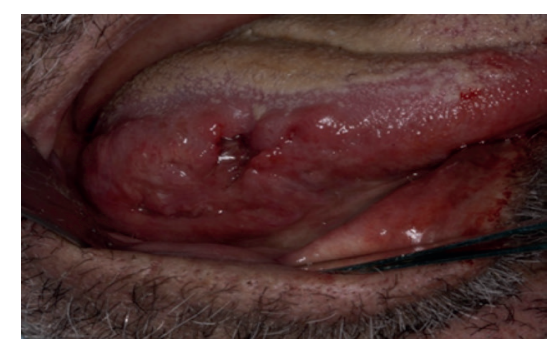

Fig. 1. Carcinoma espino celular altamente diferenciado de lengua. Lesión ulcerada en borde lateral de lengua. Paciente alcohólico y fumador crónico (01 paquete de cigarros/día durante 45 ańos).

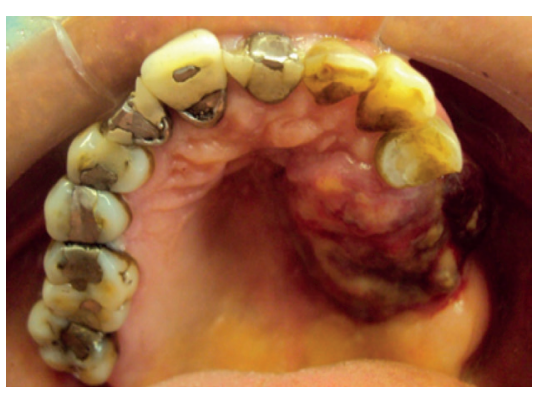

Fig. 2. Carcinoma espinocelular indiferenciado. Lesión exofítica, sésil, localizada en mucosa palatina izquierda. 


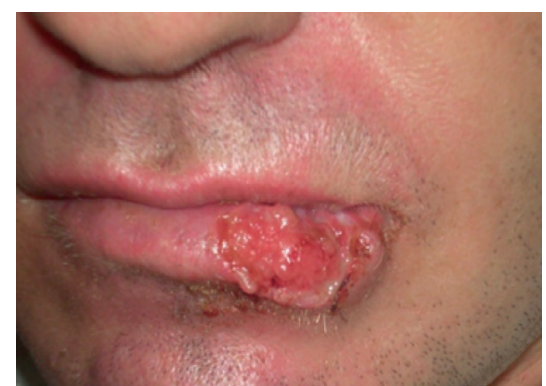

Fig. 3. Carcinoma espino celular de labio. Lesión ulcerada en bermellón de labio inferior. Paciente altamente expuesto a la radiación solar (chofer de camión) y fumador crónico. El CEC de labio tiene un mejor pronóstico, pues este no se comporta tan agresivamente como el carcinoma intraoral.

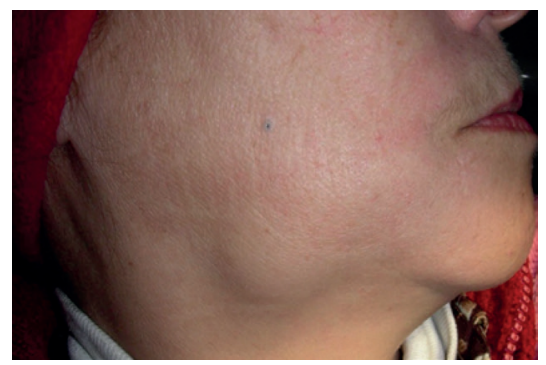

Fig. 4. Metástasis ganglionar submandibular en paciente afectada porCEC de lengua. Caso cortesía del Dr. Benjamín Martínez (patoral@umayor.cl)

\section{Agradecimientos:}

- Los autores desean expresar su agradecimiento a:

- Prof. Dra. Erika Harth-Chú por su ayuda en el desarrollo y corrección del manuscrito.

\section{Referencias bibliográficas}

1. Carvalho MB, Lenzi J, Lehn CN, et al. Características clínico-epidemológicas do carcinoma epidermóide de cavidade oral no sexo feminino. Rev. Assoc. Med. Bras. 2001; 47(3); 23-27.

2. Sankaranarayanan R, Masuyer E, Swaminathan R. Head and neck cancer: a global perspective on epidemiology and survival. Anti Cancer Res 1998; 18: 4779-86.

3. Instituto Nacional do Câncer / Ministério da Saúde. Estimativa 2008: incidência de câncer no Brasil. Rio de Janeiro: Instituto Nacional de Câncer; 2007. Disponível em URL: http://www.inca.gov.br

4. Cooper JS, Pajak TF, Forastiere AA, et al. Postoperative concurrent radiotherapy and chemotherapy for high-risk squamous-cell carcinoma of the head and neck. N Engl J Med 2004; 350:1937-1944.

5. Scully C, Felix DH. Oral medicine-update for the dental practitioner oral cancer. Br Dent J. 2006; 200(1): 13-7.

6. Sklenicka S, Gardiner S, Dierks EJ, Potter BE, Bell RB Survival analysis and risk factors for recurrence in oral squamous cell carcinoma: does surgical salvage affect outcome? J Oral Maxillofac Surg. 2010; 68(6): 1270-5.

7. Mansour OI, Snyderman $\mathrm{CH}$, D'Amico F. Association between tobacco use and metastatic neck disease. Laryngoscope. 2003; 113 (1): 161-6.

8. Neville BW, Damm DD, Allen CM, Bouquot J. Oral \& Maxillofacial Pathology. 2. ed. Philadelphia: W. B. Saunders Company; 2002. p. 578-580.

9. Rosenquist K. Risk factors in oral and oropharyngeal squamous cell carcinoma: a population-based case-control study in southern Sweden. Swed Dent J Suppl. 2005; (179): 1-66.

10. Leão JC, Góes P, Sobrinho CB, Porter S. Knowledge and clinical expertise regarding oral cancer among Brazilian dentists. Int J Oral Maxillofac Surg. 2005; 34(4): 436-9.

11. Macfarlane GJ, Boyle P, Scully C. Oral cancer in Scotland: changing incidence and mortality. BMJ. 1992. 7; 305(6862): 1121-3.

12. Zelefsky M, Harrison L, Fass D, et al. Post operative radiation therapy for squamous cell carcinoma of the oralcavity and oral pharynx: impact of therapy on patients with positive surgical margins. Int J Rad Biol Phys 1992; 25: 17-21.

13. Leemans C, Tiwari R, Nauta J, et al: Recurrence at the primary site in head and neck cancer and the significance of neck lymph node metastasis as a prognostic factor. Cancer, 1994; 73: 187.

14. Regezi JA, Sciubba, JJ. Oral Pathology - clinical pathologic correlations. 3. ed. Philadelphia: W.B. Saunders Company. 1999. p. 75-76.

15. Betka J. Distant metastases from lip and oral cavity cancer. ORL J Otorhinolaryngol Relat Spec. 2001; 63(4): 217-21
16. Shiboski CH, Schmidt BL, Jordan $\mathrm{RC}$ : Tongue and tonsil carcinoma: increasing trends in the U.S. population ages $20-44$ years. Cancer, 2005; 103: 1843.

17. Adelstein DJ, Li Y, Adams GL, Wagner $\mathrm{H}$ Jr, Kish JA, Ensley JF, et al. An Intergroup Phase III Comparison of standard radiation therapy and two schedules of concurrent chemoradiotherapy in patients with unresectable squamous cell head and neck cancer. J Clin Oncol. 2003; 21(1): 92-98.

18. Macpherson LM, McCann MF, Gibson J, Binnie VI, Stephen KW. The role of primary healthcare professionals in oral cancer prevention and detection. Br Dent J. 2003 13; 195(5): 277-81.

19. Fang FM, Chiu HC, Kuo WR, et al. Health-related quality of life for nasopharyngeal carcinoma patients with cancer-free survival after treatment. Int J RadiatOncolBiol Phys. 2002; 53: 959-968.

20. Allison P, Franco E, Feine J. Predictors of professional diagnostic delays for upper aerodigestive tract carcinoma. Oral Oncol. 1998; 34: 127-132.

21. Clovis JB, Horowitz AM, Poel $\mathrm{DH}$. Oral and pharyngeal cancer: knowledge and opinions of dentists in British Columbia and Nova Scotia. J Can Dent Assoc. 2002; 68(7): 415-20.

22. Kuriakose MA, Sharan R. Oral cancer prevention. OralMaxillofacSurgClin North Am. 2006; 18(4): 493-511.

23. Muñante-Cárdenas JL, de Assis AF, Olate S, Lyrio MC, de Moraes M. Treating oral histoplasmosis in an immunocompetent patient. J Am Dent Assoc. 2009; 140(11): 1373-6.

24. Parise Júnior O. Câncer de boca: aspectos básicos e terapêuticos. 1 . ed. São Paulo: Sarvier; 2000.

25. Silva SD, Agostini M, Nishimoto IN et al. Expression of fatty acid synthase, ErbB2 and Ki-67 in head and neck squamous cell carcinoma. A clinicopathologicalstudy. Oral Oncol 2004; 40(7): 688-96. 